Pérez-Hernández et al., Afr J Tradit Complement Altern Med., (2018) 15 (1): 168-173

https://doi.org/10.21010/ajtcam.v15i1.17

\title{
ANTIUROLITHIC ACTIVITY OF BERBERIS TRIFOLIATA EXTRACT ON INDUCED UROLITHIASIS IN RATS BY ZINC DISC IMPLANTATION
}

\section{Raymundo Alejandro Pérez-Hernández ${ }^{1}$, Silvia Guadalupe Treviño-Moreno ${ }^{1}$, Gilberto Arévalo- Martínez ${ }^{2}$, Eduardo Sánchez -García ${ }^{1}$, Catalina Leos-Rivas ${ }^{1}$, Catalina Rivas-Morales ${ }^{1 * *}$}

${ }^{1}$ Universidad Autónoma de Nuevo León, Facultad de Ciencias Biológicas, Departamento de Química, San Nicolás de los Garza, Nuevo León, México. ${ }^{2}$ Universidad Autónoma de Nuevo León, Facultad de Medicina, Departamento de Fisiología, Monterrey, Nuevo León, México.

All authors contributed equally to this work.

*Corresponding Author E-mail: catalinarivas@yahoo.com.mx

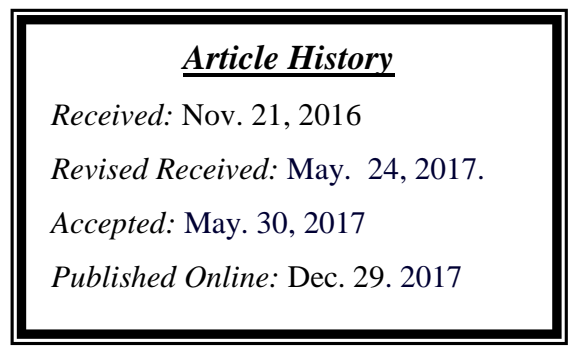

\begin{abstract}
Background: In clinical therapy, there is no satisfactory drug available for treatment of urolithiasis, especially for the prevention of their recurrence. The aim of this work was to evaluate in vivo antiurolithic activity of methanolic extract of Berberis trifoliata leaves.

Material and methods: Urolithiasis was induced in Wistar rats by zinc disc implantation in urinary bladder. Upon postsurgical recovery, different doses of the methanolic extract of $B$. trifoliata leaves $(50,100 \mathrm{and} 150 \mathrm{mg} / \mathrm{kg}$ body weight) were administered orally to zinc disc implanted rats for a period of 20 days. Antiurolithiatic activity was evaluated by measuring the difference between the weight of the implanted zinc discs at the time of implantation and the final weight of the dried calculi taken out from the bladder at the end of the 20 days period of treatment.

Results: Extract of B. trifoliata significantly reduced calculi deposition around the implanted zinc disc at all doses $(50,100$, and $150 \mathrm{mg} / \mathrm{kg}$ ).

Conclusion: Treatment with methanolic extract of B. trifoliata is useful agent against the kidney stone formation.
\end{abstract}

Keywords: Antiurolithic, Berberis trifoliata, urolithiasis, zinc disc implantation.

\section{Introduction}

Urolithiasis is a condition in which urinary calculi are formed and located at any level of the urinary system (Tiwari et al., 2012; Rajeshwari et al., 2013). Formation of stones is the third most common problem of the human urinary system. (Bashir and Gilani, 2011; Khan et al., 2011). It is a worldwide problem; it is estimated that $12 \%$ of the world population experiences renal stone disease with a high recurrence rate (Khan et al., 2011; Narendra and Ameeta, 2013). Urinary stone formation is the result from several physicochemical events including nucleation, supersaturation and crystal growth. Calcium oxalates are the primary constituent of the majority of urinary tract stones (Bangash et al., 2011). Minimally invasive surgery including extracorporeal shock wave lithotripsy (ESWL), percutaneous nephrolithotomy (PCNL) or ureteroscopy (URS) are considered effective removal techniques, but they are costly, making them an limited option and data suggest that these techniques have some side effects (Khan et al., 2012). Complications include residual stone fragments as potential nidus for new stone formation, compromised renal function, acute renal injury and urinary tract infection. Despite of advancements in the pathophysiology and treatment modalities of urolithiasis, there is no satisfactory drug and medical treatment available, especially for the prevention of stones formation recurrence (Padma et al., 2016). 
There is growing public interest in herbal medicine, particularly in the management of urolithiasis. In this study, we evaluated aerial part of $B$. trifoliata for in vivo antiurolithic activity. Berberis trifoliata Moric (also known as Mahonia trifoliata) is a plant that belongs to the barberry family Berberidaceae populary known as agrito, algerita and desert holly. B. trifoliata can be found growing from 4000 to $7000 \mathrm{ft}$. in elevation in central, west and north Texas; New Mexico, Arizona and Mexico. This plant is a popular folk remedy in the south of the Nuevo León, Mexico for the treatment of urinary tract and renal disorders including kidney stones. For this purpose, the natives use medicinal tea from the fresh stem.

\section{Materials and methods Plant material}

Leaves of Berberis trifoliata growing wild were collected in March and April in the community of Casa Blanca,

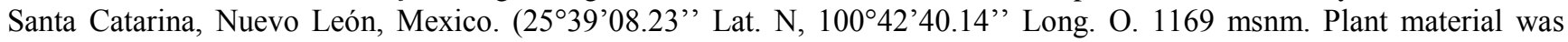
deposited at the herbarium of Facultad de Ciencias Forestales of Universidad Autonoma de Nuevo Leon, (FCNL), for taxonomic identification and voucher number 0100 was assigned for reference at the herbarium of this institution.

\section{Extraction}

The plant material was shade dried at room temperature $\left(25 \pm 2^{\circ} \mathrm{C}\right)$ and then triturated using a commercial hand mill (Victoria). The extraction was performed with methanol using maceration method with constant shaking at room temperature during 48 hours. After that, the extract was filtered through Whatman filter paper twice. The final filtrate was evaporated to dryness in a rotary evaporator (Yamato Scientific CO. LTD. RE 200) under controlled temperature and pressure to obtain the crude extract. The dried extract was stored in airtight container and kept in refrigeration at $4^{\circ} \mathrm{C}$ until used.

\section{Animals}

For this study a total of thirty male Wistar rats of 10-12 weeks old and 300-350 g body weight were selected. All animals were kept in plastic cages and maintained under controlled conditions including: $12 \mathrm{~h}$ of dark and light cycle, room temperature of $21 \pm 2{ }^{\circ} \mathrm{C}$ and 50-55\% relative humidity. Animals were fed with standard rat chow diet (commercially available standard pellet feed; Prolab laboratory animal diet, St. Louis, MO, USA). Animals had free access to food and water ad libitum throughout the study. The animals were maintained under laboratory conditions for 7 days before the commencement and during the experiment. All procedures were performed according to the ethical standard guidelines for the care and use of animals in scientific research (NOM-062-ZOO-1999).

\section{Surgical implant of zinc disc}

Before the implantation, 36 zinc discs with an average weight of: $18.2 \pm 0.2 \mathrm{mg}$ were prepared and autoclave sterilized. The zinc disc implantations in urinary bladders were carried out according to an earlier reported method (Pawar and Vyawahare, 2016). Prior to anesthesia, to dilate their urinary bladders, rats were orally administered with $4 \mathrm{~mL}$ of purified water. Rats were operated in sterile conditions and anesthetized under ketamina (Clorkétam ${ }^{\circledR} 1000$, Vétoquinol laboratories, Mexico) (10 mg/kg intraperitoneally) and xilacina (Procin®, PiSA Laboratories, Mexico) (3 mg/kg intramuscularly). When the reflexes were abated, the urinary bladder was exposed through a suprapubic incision, the urine was aspirated with sterile syringe and a small cut was taken to open the lumen of the bladder. Finally, one zinc disc weighting $18.4 \pm 0.2 \mathrm{mg}$ was inserted into the bladder and the incision was closed by suturing with absorbable sterile surgical sutures (VICRYL ${ }^{\text {TM }}$ 5-0 USP). The urinary bladder was pushed back into its original place. The muscular layer of the abdomen and the skin incision were separately sutured. When the animals recovered from anesthesia, as a postsurgical analgesic, tramadol (Tramadol Jet, NORVET laboratories, Mexico) $(30 \mathrm{mg} / \mathrm{kg}$ ) and sodic metamizol (Prodol-Jee NRV*, NORVET laboratories, Mexico) $(10 \mathrm{mg} / \mathrm{kg})$, were administered orally and this analgesic therapy was repeated every $12 \mathrm{~h}$ for 3 days. All the operated rats were treated with the antibiotic enrofloxacin (Baytril $5 \%$, Bayer) $(1 \mathrm{ml} / \mathrm{kg}$ ) and allowed to recover for 3 days.

\section{Experimental groups}

The rats were divided into five treatment groups of 6 each: Group I: vehicle-treated control, Group II: Experimental Control (only zinc disc implanted), Group III: zinc disc implanted and treatment with plant extract (50 $\mathrm{mg} / \mathrm{kg} /$ day, p.o.), Group IV: zinc disc implanted and treatment with plant extract (100 mg/kg/day p.o.), Group V: zinc disc 
implanted and treatment with plant extract (150 mg/kg/day p.o.). The plant extract was dissolved in $0.9 \%$ Normal Saline and the treatment duration was for 20 days.

\section{Radiographical studies}

$\mathrm{X}$-rays on rats were taken for monitoring the crystal deposition in the bladders of zinc disc implanted rats on X-ray machine (The Weber dental MFG, Co, Canton, Ohio, USA).

\section{Weight of the formed stone}

After 20 days of treatment, all animals were euthanized by deep anesthesia. The urinary bladders were exposed and the zinc discs with the adhered crystals were removed. The difference between the weight of the implanted zinc discs at the time of implantation and the final weight of the dried calculi taken out from the bladder at the end of the 20 days period indicates the amount of deposited stone.

\section{Statistical Analysis}

Experimental results were reported as means \pm standard error of mean (SEM) and 95\% confidence limits (CL). Statistical analysis was performed by SPSS software (Statistical Package for the Social Science, version 20.0, SPSS Inc, Chicago, Illinois, USA), using one-way analysis of variance (ANOVA) followed by the Dunnett post-hoc test for determining the statistical significance of the difference in the weights of the deposits around the implanted discs between the treated groups and the corresponding zinc disc-implanted control group. The mean difference was significant at the $\mathrm{p}<0.01$ level.

\section{Results}

The yield of methanolic extract of Berberis trifoliata was found to be $5.40 \% \mathrm{w} / \mathrm{w}$. The results obtained from the antiurolithic activity assay are shown in figure 1. At the end of 20-day period, in the vehicle-treated control group, there was no such deposition in the bladder lumen. On the other hand, in the only zinc disc implanted group without any treatment, zinc disc implantation caused deposition of calculi around the implanted disc, the average weight of deposited crystals was found to be $94.62 \pm 12.01 \mathrm{mg}$. In treated groups with 50,100 and $150 \mathrm{mg} / \mathrm{kg} / \mathrm{day}$ p.o. of plant extract, the formation of urinary bladder stones was inhibited noticeably. When the effect of various treatments on the weight of stones was compared to the control, it was observed that the treatment of rats with alcoholic extract of $B$. trifoliata significantly reduced the weight of the depositions around the implants, (figure 2). There was no significant difference between the weights of the bladder content in the treated groups. As shown in Figure 3, the deposition around the implanted discs in different study groups was monitored by X-ray examination. 


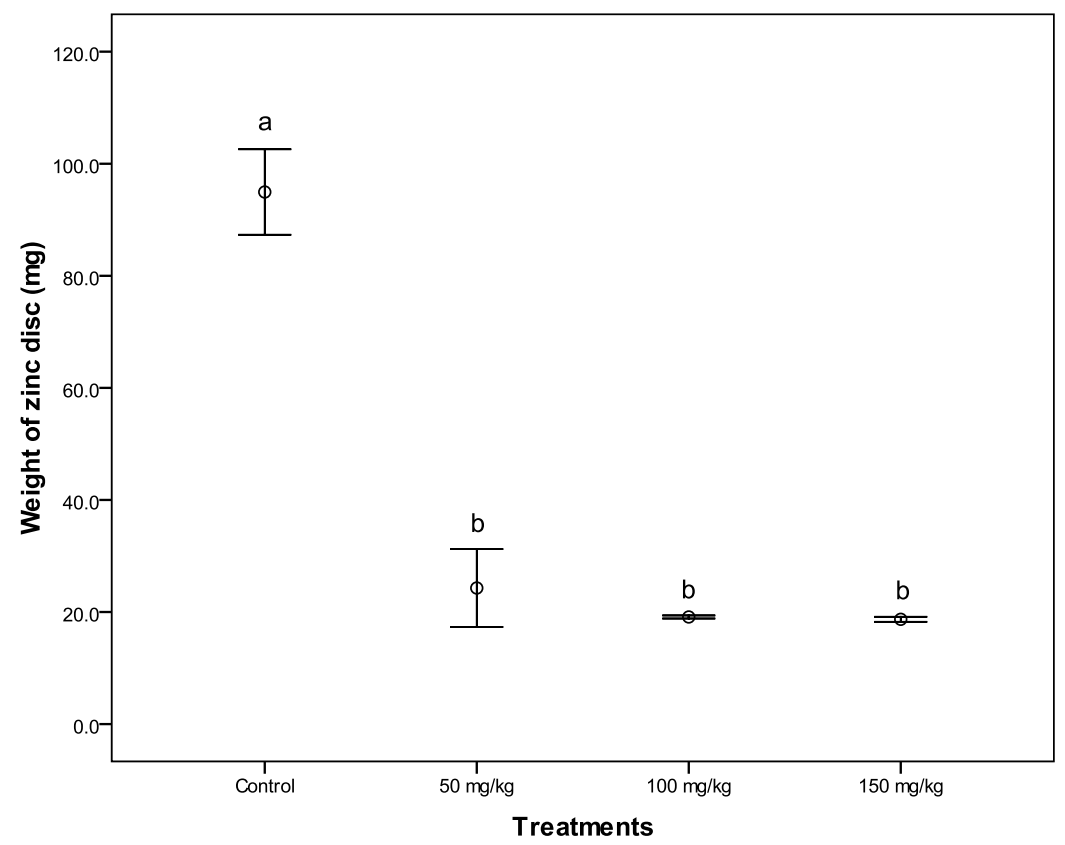

Figure 1: Descriptive statistics (mean \pm standard error) deposition of crystals in the zinc disc. $(\mathrm{F}=206.50, \mathrm{p}<0.01)$. There is no significant difference between means have the same letter.


Figure 2: Bladder stones obtained from the bioassay. (a) Experimental Control (only zinc disc implanted), (b) zinc disc implanted and treatment $50 \mathrm{mg} / \mathrm{kg}$ p.o., (c) zinc disc implanted and treatment $100 \mathrm{mg} / \mathrm{kg}$ p.o., (d) zinc disc implanted and treatment $150 \mathrm{mg} / \mathrm{kg}$ p.o.

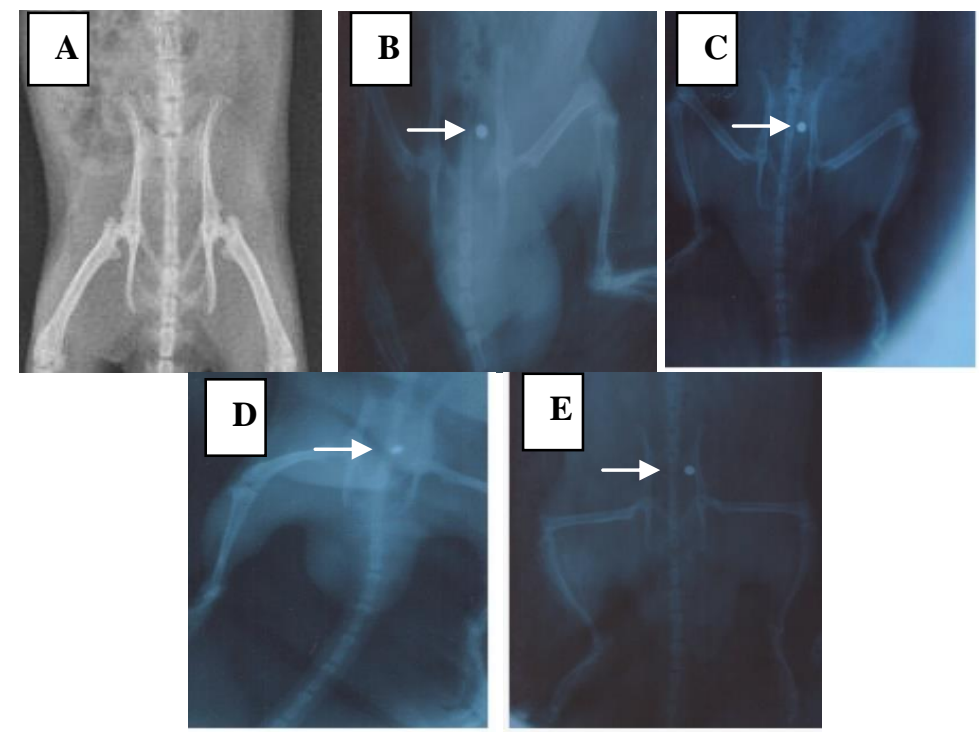

Figure 3: X-ray images of antiurolithic activity of B. trifoliata extract. (a) vehicle-treated control, (b) Experimental Control (only zinc disc implanted), (c) zinc disc implanted and treatment $50 \mathrm{mg} / \mathrm{kg}$ p.o., (d) zinc disc implanted and treatment 100 $\mathrm{mg} / \mathrm{kg}$ p.o., (e) zinc disc implanted and treatment $150 \mathrm{mg} / \mathrm{kg}$ p.o. 


\section{Discussion}

Extraction procedures play a significant and crucial role on the qualitative and quantitative studies of bioactive compounds (Azmir et al., 2013). A range of techniques may be used for extraction of plant material such as maceration, Soxhlet extraction, microwave-assisted (MAE), ultrasound-assisted extraction (UAE) and supercritical fluid extraction (SFE) (Azwanida, 2015). Maceration has been widely used in medicinal plant research to extract valuable bioactive compounds. This technique is simple, and in contrast to other techniques, in which heat is used, this method avoids thermal decomposition of bioactive compounds (Handa et al., 2008; Heinrich et al., 2012). Also, it is a technique that allows implementing shaking. This facilitates the extraction by increasing the diffusion, and removes concentrated solution from the sample surface bringing new solvent to the menstruum for more extraction yield (Azmir et al., 2013).

The preclinical evaluation of newly drugs with antiurolithiatic activity are usually carried out in experimental models that mimic all the processes involved in the urinary stone disease (Singh et al., 2010). The in vitro models simulate the different phases involved in the urolith formation such as nucleation, crystal growth and crystal aggregation. However, in vitro models do not encompass the effects of endogenous stone promoters or inhibitors and biological processes that are actually involved in the stone formation (Biyani et al., 2007). Hence, in the evaluation of the antiurolithiatic effect of test drugs, in vivo animal models are preferred. Various animal models have been employed to induce urolitiasis for investigating stone formation and the effect of various therapeutic agents on the development and progression of urolitiasis (Liu et al., 2007; Narendra and Ameeta, 2013).

For this purpose, rats are generally used as experimental animals because their urinary system has structural and physiological similarities the human urinary system (Shukla et al., 2014). Renal calculi formation can be induced experimentally by the administration of various chemicals or by the surgical insertion of a foreign body into the bladder of the experimental animal models (Singh et al., 2010). Calculi are chemically induced by the oral administration of such as ethylene glycol, oxalate, gentamicin sulfate, glycolic acid, ammonium chloride, or L-hydroxyproline (Oh et al., 2011). However, these models are associated with nephrotoxicity and hence, may not represent the actual disease as it appears clinically (Laikangbam and Damayanti, 2012). Models of surgical implantation materials like zinc discs, calcium oxalate crystals, plastic discs and pieces of non-absorbable surgical threads can be implanted in the urinary bladder to induce urolithiasis. Zinc disc implantation model induces urinary calculi without severe renal damage, and is used to mimic the etiology of urinary stone formation in humans (Narendra and ameeta, 2007). Therefore, it is the most used and has been more extensively reported in the scientific literature (Singh et al., 2010). In this study, male rats were selected to induce urolithiasis because earlier studies have shown that the amount of stone deposition in female rats was significantly less (Patel et al., 2012). In females, the estrous cycle can profoundly modify certain biological responses. In the follicular phase, just before ovulation, there is an increase in estrogen levels. This triggers the activation of stress inducing mechanisms with the consequent physiological impact on organic responses and functions. For this reason, the experimental response may be clearly different in females than in males.

In the present study, all animals in the control group developed crystal depositions at the end of 20 days postimplantation. In the treated groups a dose-dependent inhibitory effect of $B$. trifoliata on urinary bladder stone formation by the implanted zinc discs was observed. Administration of the plant extract (50,100 and $150 \mathrm{mg} / \mathrm{kg} / \mathrm{day}$ for 20 days) to implanted rats, prevented the formation of urinary stones. The extract did not produce change in the spontaneous motor activity or signs of toxicity up to 20 days of administration. The formation of calculus in urinary bladder was also confirmed by X-ray. Various physiological inhibitors of urolithiasis were found in urine including citrate, glycosaminoglycans and other macromolecules. Interference with crystal growth and aggregation therefore seems a possible therapeutic strategy for the prevention of recurrent stone disease. The medicinal plants contain chemical compounds which themselves possess an inhibitor effect in the crystallization (Bensatal and Ouahrani, 2008). The genus Berberis in traditional and folklore medicine has been used for its many pharmacological and biological activities, which make it an effective alternative to primary medical treatment of various diseases. Phytochemical analysis revealed the presence of alkaloids, tannins and phenolic compounds (Mokhber-Dezfuli et al., 2014; Srivastava et al., 2015). One of the main compounds, found in various species of genus Berberis is berberine that has been identified as the main responsible natural compounds for diverse therapeutic potentials. Plants rich in berberine have broad medicinal applications in virtually all traditional systems and have several therapeutic uses in common including antiurolithic activity (Bashir and Gilani, 2011). We suggested that the extract probably contains alkaloid berberine that prevents urinary stone formation by excretion of small particles from the kidney and reduce the chance of them being retained in the urinary tract.

\section{Conclusions}

The treatment with methanolic extract of $B$. trifoliata in zinc disc implanted rats produced a significant decrease in the weight of calculi in the urinary bladder. The results from the present study confirmed that the extract contained antiurolithic agents and the utility of use of the plant in folk medicine against urolithiasis. 
Conflict of Interest: Authors declare that they have no conflicts of interest.

\section{References}

1. Azmir J., Zaidul I.S.M., Rahman M.M., Sharif K.M., Mohamed A., Sahena F., Jahurul M.H.A., Ghafoor K., Norulaini N.A.N., Omar A.K.M. (2013). Techniques for extraction of bioactive compounds from plant materials: A review, J. Food Engr., (4)117, 426-436, ISSN 0260-8774, http://dx.doi.org/10.1016/j.jfoodeng.2013.01.014.

2. Azwanida NN. (2015). A Review on the Extraction Methods Use in Medicinal Plants, Principle, Strength and Limitation. Med Aromat Plants 4:196. doi:10.4172/2167-0412.1000196.

3. Bangash K, Shigri F, Jamal A, Anwar K (2011) Spectrum of renal stones composition; chemical analysis of renal stones. Int J Pathology 9: 63-66.

4. Bashir S \& Gilani AH. (2011). Antiurolithic effect of berberine is mediated through multiple pathways, Eur J Pharmacol 651(1-3) 168.

5. Bensatal, A., \& Ouahrani, M. R. (2008). Inhibition of crystallization of calcium oxalate by the extraction of Tamarix gallica L. Urol res 36(6), 283-287.

6. Biyani CS, Basavaraj DR, Anthony JB, Jon JC. (2007). The role of urinary kidney stone inhibitors and promoters in the pathogenesis of calcium containing renal stones. EAU-EBU Update Series. 5:126-36.

7. Handa SS, Khanuja SPS, Longo G, Rakesh DD. (2008). Extraction Technologies for Medicinal and Aromatic Plants, (1stedn), no. 66. Italy: United Nations Industrial Development.

8. Heinrich, M., Barnes, J., Gibbons, S., \& Williamson, E. M. (2012). Fundamentals of pharmacognosy and phytotherapy. Elsevier Health Sciences.

9. Khan A, Bashir S, Khan SR, Gilani AH. (2011). Antiurolithic activity of Origanum vulgare is mediated through multiple pathways. BMC Complement Altern Med. 11:96.

10. Khan, A., Khan, S. R., Anwar, H. Gilani. (2012). Studies on the in vitro and in vivo antiurolithic activity of Holarrhena antidysenterica, Urol Res. 40(6):671-81.

11. Laikangbam R, Damayanti Devi M. (2012). Inhibition of calcium oxalate crystal deposition on kidneys of urolithiatic rats by Hibiscus sabdariffa L. extract. Urol Res 40(3):211-8.

12. Liu J, Cao Z, Zhang Z, Zhou S, Ye Z. (2007). A comparative study on several models of experimental renal calcium oxalate stones formation in rats. J Huazhong Univ Sci Technolog Med Sci. 27(1), 83-87.

13. Mokhber-Dezfuli, N., Saeidnia, S., Gohari, A. R., \& Kurepaz-Mahmoodabadi, M. (2014). Phytochemistry and pharmacology of berberis species. Pharmacogn rev, 8(15), 8.

14. Narendra Vyas and Ameeta Argal. (2013). Antiurolithiatic Activity of Extract and Oleanolic Acid Isolated from the Roots of Lantana camara on Zinc Disc Implantation Induced Urolithiasis. ISRN Pharmacol. 2013:951795. doi: $10.1155 / 2013 / 951795$.

15. Oh SY, Kwon JK, Lee SY, Ha MS, Kwon YW, Moon YT. (2011). A comparative study of experimental rat models of renal calcium oxalate stone formation. 25(6):1057-61. doi: 10.1089/end.2010.0386.

16. Padma Nibash Panigrahi, Sahadeb Dey, Subash Chandra Jena. (2016). Urolithiasis: Critical Analaysis of Mechanism of Renal Stone Firmation and Use of Medicinal Plants as Antiurolithiatic Agents. Asian J Anim Vet Adv. 11: 9-16. DOI: 10.3923/ajava.2016.9.16

17. Patel PK, Patel MA, Vyas BA, Shah DR, Gandhi TR. (2012). Antiurolithiatic activity of saponin rich fraction from the fruits of Solanum xanthocarpum Schrad. \& Wendl. (Solanaceae) against ethylene glycol induced urolithiasis in rats.J Ethnopharmacol. Oct 31;144(1):160-70. doi: 10.1016/j.jep.2012.08.043.

18. Pawar AT, Vyawahare NS. (2016). Antiurolithiatic activity of Abelmoschus moschatus seed extracts against zinc disc implantation-induced urolithiasis in rats. J Basic Clin Pharm. 7(2):32-38.

19. Rajeshwari P, Rajeswari G, Jabbirulla SK, and Vishnu Vardhan I. (2013). Evaluation of in vitro antiurolithiasis activity of Convolvulus arvensis. Int J Pharm Pharm Sci. 5(3): 599-601.

20. Shukla AB, Mandavia DR, Barvaliya MJ, Baxi SN, Tripathi CR. (2014). Evaluation of anti-urolithiatic effect of aqueous extract of Bryophyllum pinnatum (Lam.) leaves using ethylene glycol-induce renal calculi. Avicenna $\mathbf{J}$ Phytomed. 4(3):151-159.

21. Singh PK, Patil CR, Harlalka GV, Gaud NP. (2010). Zinc disc implantation model of urinary bladder calculi and humane endpoints. Lab Anim. 44(3):226-30.

22. Srivastava S, Srivastava M, Misra A, Pandey G, Rawat A. (2015). A review on biological and chemical diversity in Berberis (Berberidaceae). EXCLI J. 20;14:247-67.

23. Tiwari A, Soni V, Londhe V. (2012). An overview on potent indigenous herbs for urinary tract infirmity: urolithiasis. Asian J Pharm Clin Res; 5(1):7-12. 